\title{
Improved first and second order control in tip edge and preadjusted edgewise appliance: KD ligation technique
}

\author{
Shweta R Bhat ${ }^{1, *}$, Kartik Dholakia ${ }^{2}$, Aditya Talwar ${ }^{3}$ \\ ${ }^{1}$ Professor and Head, ${ }^{2}$ Private Practitioner, ${ }^{3} \mathrm{PG}$ student, Nair Hospital Dental College, Mumbai
}

*Corresponding Author:

Email: srbhat25@outlook.com

\begin{abstract}
Tip edge brackets have evolved from the edgewise brackets with the removal of the diagonally opposite corners to incorporate the features of the Begg's philosophy. Consequently, with the increase in the versatility of the brackets, additional auxillaries were required during the finishing stage of the orthodontic treatment. With different malocclusions having different requirements, it strains the limit for which the brackets are designed. Thus, it comes to the versatility of orthodontist skills to device techniques to use the full potential of the brackets while achieving the treatment objectives in the most efficient way. In the present case report, we have enumerated the versatility of ligation techniques which can be used to suit the needs of the case and achieve the correction of the malocclusion in the most efficient way.
\end{abstract}

Keywords: Tip edge brackets, Ligation technique, First and Second Order control, Preadjusted Edgewise Brackets.

\section{Introduction}

Tip Edge brackets ${ }^{1}$ developed by Peter Kesling was modified from the standard edgewise bracket by removal of diagonally opposite corners to facilitate the initial tipping movement of the teeth. ${ }^{1}$ This modification in the bracket system harnessed the benefits of tipping present in Begg's technique involving the differentiation of root and crown movement forces, while maintaining inbuilt rotational and torque control of Edgewise Appliance system. Consequently, with the development of the Tip Edge PLUS bracket series, an additional deep tunnel is introduced to the conventional design. This deep tunnel when used to thread 0.016 thermal NiTi in the third stage of the treatment maintains the rotational corrections, enhance tip expression and subsequent root uprighting, thus providing the functionality of sidewinder spring and making the third stage 'spring free stage'. ${ }^{2}$

The objectives of the three stages of Tip Edge treatment philosophies are achieved by exploiting specific design characteristics of brackets in addition to the use of various auxillaries. Thus, tipping surfaces of Tip edge brackets have a dominant role in first two stages, however, even in the finishing stages, these surfaces play a major role. However, despite relatively wider dimensions of tip edge brackets, the rotational control is still poorer as compared to Preadjusted Edgewise brackets. Additionally Tip edge PLUS ${ }^{1}$ brackets, though, claims precludes the use of sidewinder springs for uprighting roots, even when thermally activated wires of optimum performance are used, the residual activation at the end of root uprighting is questionable. ${ }^{3}$ Also, in cases involving reinforcing of anchorage in the lower incisors, it may be desirable for the operator that the tip edge bracket performs similar to PEA bracket, by boosting the activation of springs (hyper-activated), so as to maintain a bodily controlling force after a tooth has reached its finished position. This requires the use of sidewinder springs, which, not only is unpleasing to the patient but also leads to esthetics concerns and adds to the inventory.

The present article describes the use of various innovative ligation techniques to facilitate, enhance or restrict particular tooth movement, in $1^{\text {st }}$ and/ or $2^{\text {nd }}$ order tooth movement. These ligation techniques can be selectively used on particular teeth, at any stage of treatment to enhance the treatment outcome and also decrease the number of auxillaries required for the select appliance system.

\section{Salient Features}

1. Enhanced control over tooth movements in $1^{\text {st }}$ and $2^{\text {nd }}$ order from initial stages of treatment.

2. Enhanced variable anchorage on basis of ligation technique.

3. Bite opening efficiency can be moderately increased even with $\mathrm{NiTi}$ archwires

4. No extra inventories required, therefore no additional cost.

5. Precludes use of auxillaries like sidewinder springs, thereby reducing the cost and the unpleasing esthetics attributed to auxillary wires.

6. Precludes bracket repositioning for moderate root uprighting procedures, even in preadjusted bracket systems.

7. Not a difficult technique to learn.

Ligation Techniques: Three types of ligation techniques; viz Type A, Type B, and Type C, have been explained below.

Type A: For enhanced rotation control (Fig. 1): The rotation correction efficiency of Tip Edge PLUS brackets is poor in comparison to Preadjusted edgewise (PEA) brackets, despite increased overall width Tip 
Edge brackets. The reason for this decreased efficiency is due to relatively shorter distance between the tie wings used to ligate archwire in the bracket slot $(\sim 2$ $\mathrm{mm}$ in Tip Edge PLUS Rx 1 bracket, as compared to $6.35 \mathrm{~mm}$ to $3.18 \mathrm{~mm}$ in Preadjusted edgewise (PEA) bracket, for upper central incisors and canine/ premolar bracket respectively), thus decreasing the amount of couple generated for correction of rotations $\mathrm{s}^{4}$.

Procedure: It involves the use of a deep tunnel of the bracket on the tooth requiring rotational correction. The two ends of stainless steel ligature wire $\left(0.008^{\prime \prime}\right)$ (preferably pre-stretched) is passed over the archwire and threaded into one of the ends of the deep tunnel (preferably at the side of rotation), and pulled out from the other end. The two ends of ligature wire are then passed over the archwire and ligated (Fig. 1), such that archwire is completely engaged in the bracket slot. This ligation method can also be used in conjunction with resilient heat treated stainless steel wire like A.J. Wilcock wire for relatively minor rotation corrections. It needs an emphasis that the tip expressed with this ligation technique is the same as that of the appliance. The effective increase in the width of the ligating ends is by approximately $2.4 \mathrm{~mm}$ (length of deep tunnel i.e. $\sim 4.4 \mathrm{~mm}$, minus the width of tie wings i.e. $\sim 2 \mathrm{~mm}$ ), thereby increasing the effective width of ligation by $120 \%$ of the width of tie wings used in conventional ligation. Thus, for a given force, the amount of two equal and opposite moments generated for rotation correction of the tooth increases by more than double, as compared to conventional ligation technique using tie wings.

This technique however needs a cautious use in cases which may oppose the basic philosophy of tip edge appliance, for examples, in presence of distally tipped and rotated canine, use of this technique may attain better rotation correction of canine, but at the expense of further bite deepening, and thus going against one of the desirable features of using Tip Edge appliance, unless required for that particular case. Therefore, this technique should be used such that it enhances rotation correction efficiency of the bracket without having significant undesirable effects on other features of the appliance system.

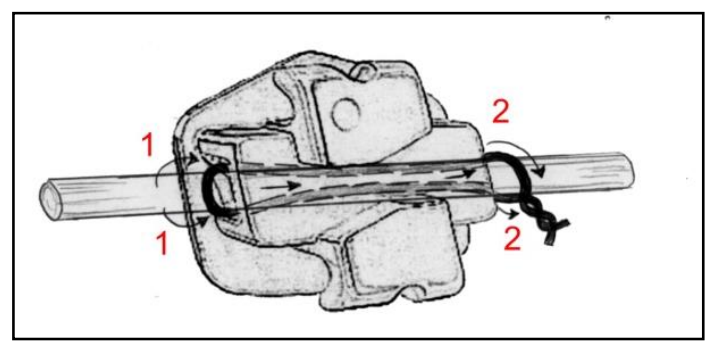

Fig. 1: Type 1 ligation technique. The numbers (in red) and arrows indicate the direction and sequence of threading the ligature wire. Dotted lines indicate the length of ligature wire within the deep tunnel of Tip Edge PLUS bracket

\section{Indications}

1. Any rotated teeth with near-normal angulations.

2. Any rotated teeth with crown mesially tipped in segment anterior to the extraction site.

3. Rotated and distally tipped teeth posterior to the extraction site.

4. Rotated and mesially tipped canine favoring bite opening, if required

Type B: For Enhance tip control along with rotation control (Fig. 2): Tip edge brackets permits up to $20-$ 25 degrees of crown tipping, attributable due to the amount of angulations between 'tip - limiting surfaces' and 'finishing surfaces'. During Stage I and II, the crown is free to tip till the tip limiting surfaces come in contact with archwire while during Stage III, teeth are uprighted till the finishing surfaces contact the archwire. These surfaces thus have their function related to the specific stage of the treatment.

With Type B ligation technique, the amount of tip that can be expressed at any stage on a particular tooth which can be suitably manipulated up to 25 degrees. Thus, with this type of ligation technique, the clinician can effectively increase/ decrease tip expression on a particular tooth depending upon the need of the treatment.

Procedure: A stainless steel ligature $\left(0.008^{\prime \prime}\right)$ (preferably prestretched) is passed through the deep tunnel, following which, one of the ends (mesial or distal depending upon which end of the archwire need to be deflected gingivally), is passed over the wire into the opening of the vertical slot from the gingival end. (Fig. 2A). The other end of the ligature wire is then passed over the archwire and ligated with the free occlusal end tightly.

The above technique of ligation helps to maintain the archwire abutting the finishing surface. (Fig. 2B). Also, this technique when used in conjunction with superelastic/ resilient wire can achieve rotational correction as good as Type A method. 


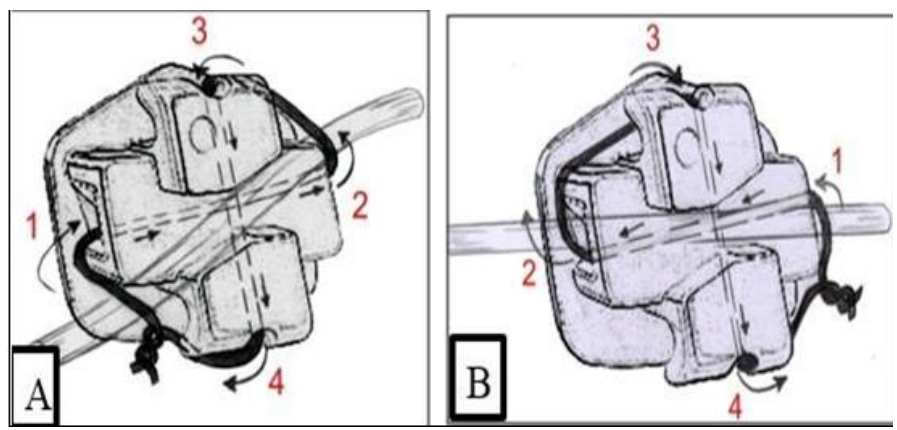

Fig. 2: Type 2 ligation method. For deflecting the anterior segment gingivally against the 'tipping surface' (A). For approximating the archwire against the 'finishing surface' (B). The numbers (in red) and arrows indicate the direction and sequence of threading the ligature wire. Dotted lines indicate the length of ligature wire within the deep tunnel and vertical slot of Tip Edge PLUS bracket

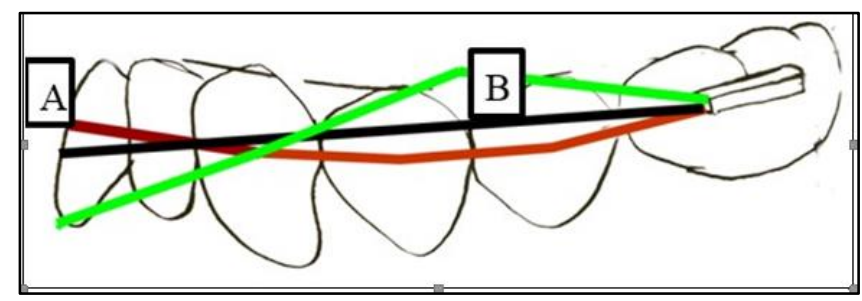

Fig. 3: Biomechanics associated with the use of Type B ligation method on canine such that archwire maintains contact with 'tip-limiting' surface. Redline: NiTi Archwire engaged in Tip edge Brackets. Black Line: NiTi arch in equilibrium. Green Line: Archwire ligated in mesially angulated canine with 'Type B' Ligation technique such that it maintains contact with 'tip limiting' surface. Note the archwire deflection (in green line), which favors bite opening efficiency along with mesial root uprighting of distally tipped canine

\section{Type B Method}

1. To enhance bite opening efficiency. (Fig. 3)

2. To correct tooth angulations, especially in Stage III, where residual activation of thermally activated NiTi archwire in the terminal phase of root uprighting is questionable.

3. For rotational correction.

4. Reinforce anchorage (or breaking mechanics)

Type C: For Enhanced tip control: Type C method of ligation using elastomeric or stainless steel ligatures can be employed in both Tip Edge and Tip Edge PLUS brackets, unlike the earlier mentioned techniques. Even though, this method does not provide the enhanced rotational control as in Type A and Type B method. Type C - E method: Use of Elastomeric rings (Fig. 4).

This technique requires threading of elastomeric rings in the form of Fig. of 8 twist from distal ends of the archwire, followed by sliding the archwire through molar tubes. Following this, the elastomeric ligature is moved along the archwire such that it lies in the region of the bracket to be ligated (Fig. 4 A) (Prestretching of the ring may be required to facilitate easier insertion and placement on the brackets).

For deflecting anterior segment of archwire gingivally: The mesial portion of Fig. of 8 elastomeric ligatures is stretched to pass over the gingival tie wing and distal portion to pass over occlusal tie wing (Fig. 4 B). And vice verse can be done for deflecting the anterior segment of the archwire incisally or maintaining the archwire contact with finishing surfaces.

Type C - S: Use of Stainless Steel Ligatures (or elastic thread): For deflecting anterior segment gingivally: With the archwire engaged in the bracket slot, the ligature wire is passed beneath the archwire on the mesial side with the gingival end beneath the gingival tie wing. The occlusal end is then re-curved from occlusal end to pass from above downwards, and beneath the archwire on the distal side. The free end of the ligature wire on the occlusal end is then passed beneath the occlusal tie wing. The two free ends of the ligature wire are then tied together over the archwire. The configuration of ligature wire will be similar to that observed with an elastomeric ligature in Type C-E method (Fig. 4 B). And vice versa can be done for deflecting the anterior segment of the archwire incisally or maintaining the archwire contact with finishing surfaces.

It should be emphasized that Type B and Type C-S method, by virtue of eliminating the play between the bracket and archwire, coupled with a firm engagement 
of archwire in bracket slot also enhances torque expression on that respective tooth.

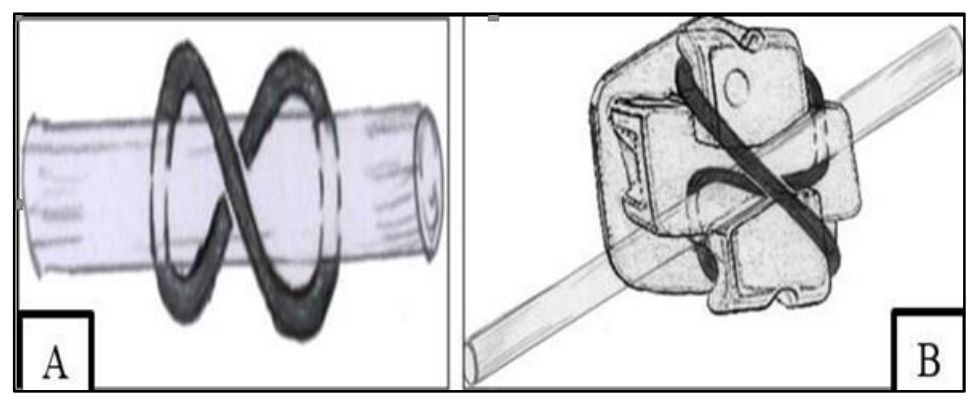

Fig. 4: Type $\mathrm{C}$ method. Elastomeric ligature threaded from the distal end of the archwire in the 'Fig. of 8' form (A). Threaded elastomeric ligature ligated to tie wings of Tip Edge bracket for deflecting the anterior portion of archwire gingivally (B). Dotted lines indicate the length of elastomeric ligature deeper to the archwire

\section{Type C method}

1. Due to its ability to completely approximate the archwire to tipping surfaces, it is better for 2 nd order of tooth movement.

2. To enhance bite opening efficiency.

3. To correct tooth angulations, especially in Stage III, where residual activation of thermally activated NiTi archwire in the terminal phase of root uprighting is questionable.

4. Reinforce anchorage (breaking mechanics).

5. Type $\mathrm{B}$ and Type $\mathrm{C}-\mathrm{S}$ method can be used in terminal phases of treatment along with resilient archwires to achieve minor root uprighting procedures. Type $\mathrm{C}-\mathrm{E}$ method can be used in to achieve root uprighting procedures even with stiffer wires, as the stretched elastomeric rings develop an inbuilt force delivery system. However, they need to be changed after every three weeks, which requires the removal of the archwire to thread new elastomeric rings.

Type B and Type C method thus preclude the use of sidewinder springs for minor root uprighting procedures.

Type $\mathrm{C}$ method may also find its application in Preadjusted Edgewise brackets, when using lighter archwires in a relatively wider bracket slot, taking an advantage of increased play between the archwire and bracket slot, the archwire can be deflected (gingivally or occlusally) with the ligation technique to correct minor tooth angulations, without the need to reposition the brackets (especially, during finishing stages of the treatment) (Fig. $5 \mathrm{~A}, \mathrm{~B}$, and $\mathrm{C}$ ).

\section{Case Report}

1. The present patient had a deep bite with a severe rotation of lower left canine (Fig. 5). For the first month of treatment, 33 was ligated with Type A method in conjunction with 0.016 " thermally activated NiTi archwire (Fig. 6A). For the following two months, tooth 33 and 44 (which was planned to function as 43 for Class I canine protected occlusion with upper canines, as 43 was horizontally impacted and extracted as a part of the treatment plan), were ligated with Type B method in conjunction with 0.016 " thermally activated NiTi archwire to favour bite opening. After only three months into the treatment, rotation of 33 was appreciable corrected (considering that 33 was placed almost in labial segment of the arch with thick cortical bone and narrow cancellous bone; rather than the buccal segment of the arch) and bite opening of approximately $3 \mathrm{~mm}$ was achieved with $0.016 \mathrm{NiTi}$ archwire (Fig. 6). Thus, it not only helped in bite opening, as well as facilitated rotation correction of canine. Also, it allowed for progression to Stage I archwires (0.016" high resilient heat treated stainless steel archwire) for bite opening, which otherwise would have not been possible in presence of rotated canine. It, therefore, reduced the treatment duration, whilst enhancing the treatment outcome. One may argue that use of the conventional sequence of archwires i.e. stage I archwires may be used initially with piggyback NiTi while waiting for complete correction of rotation of canines until later stages i.e. Stage III by threading the NiTi wire in the 'deep tunnel'. No doubt, the conventional approach could have been used in this particular case. The aim of this case report is to not prove the efficiency or possibility of use of NiTi archwire, but rather provide and prove the efficiency of the modified ligation technique. 


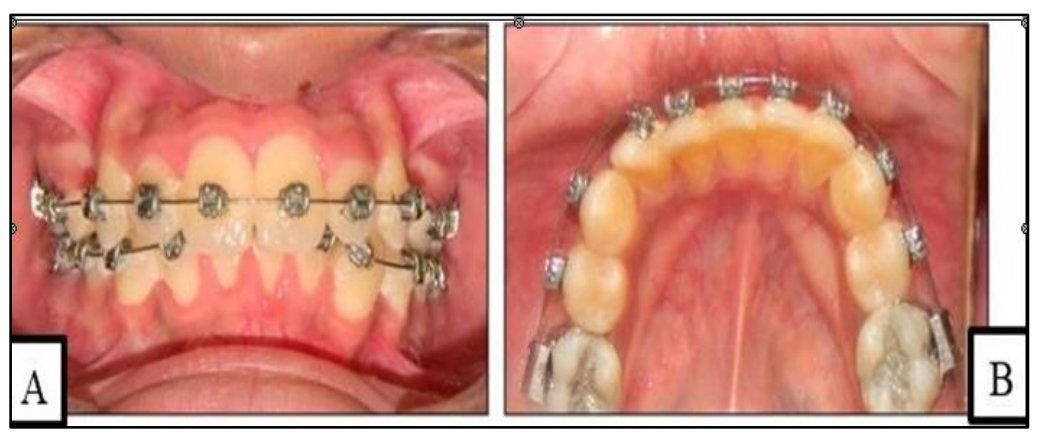

Fig. 5: Intraoral photographs before treatment. Frontal view showing severe deep bite (A). Mandibular occlusal photograph showing severely rotated $33(B)$

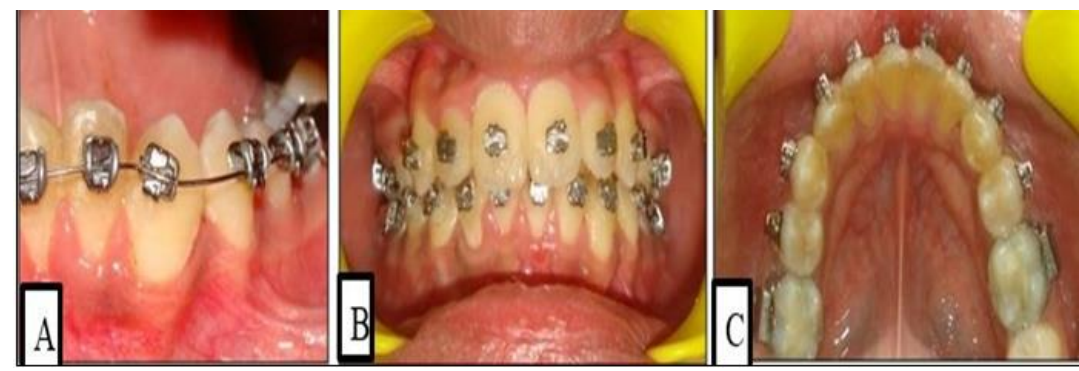

Fig. 6: Intraoral photographs after two and a half months of treatment. Frontal view (A). Mandibular occlusal photograph showing 33 after rotation correction (B)

2. Fig. 5 A shows mesially tipped 12 . The initial archwire used for alignment was $0.016 \mathrm{NiTi}$, following which 0.016 x $0.022 \mathrm{NiTi}$. In presence of conventional elastomeric ligatures (Fig. 7 A), it is observed that even with complete engagement $0.016 \times 0.022 \mathrm{NiTi}$ archwire in $0.018 "$ slot, the degree of deflection is not enough for complete uprighting of roots of 12 . With Type C-S ligation methods, the degree of deflection of $0.016 \times 0.022$ "
NiTi archwire is noticeably larger, such that it contacts the diagonally opposing corners of the bracket slot, thereby facilitating complete tip expression (Fig. 7 B). With larger size of bracket slot, there will be increased play between the archwire of $0.016 "$ so selectively facilitate tip expression/ root uprighting during finishing stages of pre-adjusted edgewise appliance.

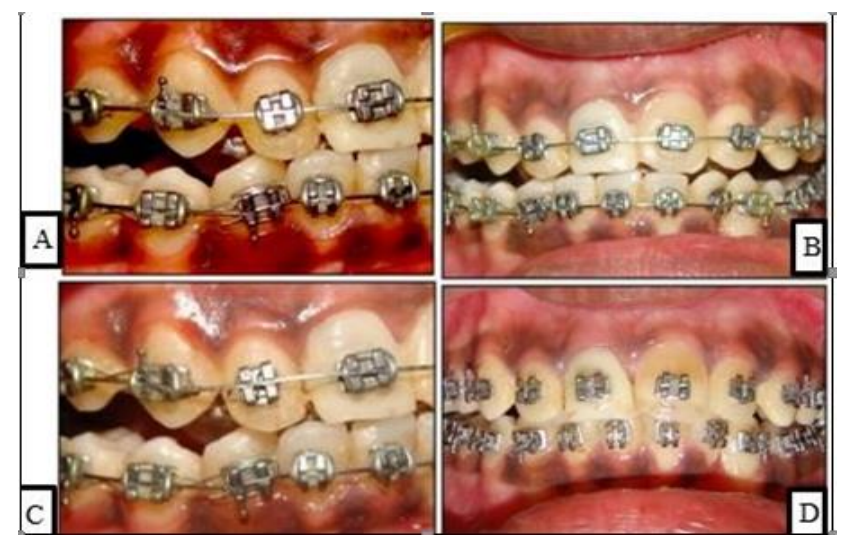

Fig. 7: 0.016 x 0.022" NiTi archwire in 0.018" bracket slot ligated with an elastomeric ligature, in relation to tooth 12 (A), and frontal photograph (B). Type B ligation method used to ligate $0.016 \times 0.022$ " NiTi archwire in the same tooth; notice the amount of archwire deflection between teeth 12 to 13 and 12 to 11 , which will lead to uprighting of tooth 12(C). After comparing with Fig. 5 (B), 12 seems uprighted after using Type B ligation technique (D). 


\section{Conclusion}

This article is aimed to widen the scope of exploiting the design features of Tip Edge/ Tip Edge PLUS able to estimate the reciprocal effects on adjacent teeth, such that it can be effectively used to perform mutually correcting tooth movements and reducing the reciprocal side effects that may arise otherwise.

Also, this modified ligation technique can be employed in conjunction with preadjusted edgewise brackets, wherein minor bracket angulation errors can be overcome during the finishing stages when undersized archwires are employed.

This method of ligation just provides an additional approach to exploit the design features of the orthodontic hardware by going beyond the boundaries of conventional methodology, but by no means is aimed to challenge the time-tested conventional methods.

\section{References}

1. Parkhouse RC. Tip-Edge Orthodontics. Edinburgh: Mosby. 2003.

2. Parkhouse RC. Tip-Edge Orthodontics and the PLUS Bracket. Second Edition.Edinburgh: Mosby. 2009.

3. Parkhouse RC. Current products and practice: Tip-Edge PLUS. J. Orthod. 2007;37(1):59-68.

4. Marcotte MR. Biomechanics in Orthodontics. B.C. Decker Inc. 1990. brackets to suit the clinicians' demand. While selectively ligating brackets with these techniques, in the presence of continuous arch wire, one should be 